\title{
Serrated flow and rupture by evolution of internal inhomogeneities
}

\author{
D. PREININGER
}

Kernforschungszentrum Karlsruhe, Institut für Materialforschung I, Postfach 3640, 7500 Karlsruhe 1, Germany

\begin{abstract}
The plastic stability and ductile rupture under evolution of cavity-damage and local strength inhomogeneities are examined theoretically for uniaxial tension. Analytical solutions are derived for that arbitrary strain-induced formations modified to stress-triaxiality evolution during necking. With increasing rate of damage formation necking is enhanced and ductility as well as load drop reduces more pronounced at large strain rate sensivities $m$ and weak initial inhomogeneities. Effects of particle concentration and external pressure deduced for nucleation controlled damage generation by particle-deponding agree with ductility observations. An inhomogeneity degradation reduces neck growth and localized flow development. Above a critical hardening rate "plastic metastability" appears with stochastic flow through structural pulsed micro-neck formation and resolution which promotes ductility. Amplitude and frequency of resulted load serrations, which quantify local structural changes correlate and depend mainly upon $\mathrm{m}$. Simultaneous multiple necking becomes stable only at superplastic or irradiation creep conditions.
\end{abstract}

\section{Introduction}

Ductile rupture of metals results by dynamic flow localization involving cavity nucleation at particles and their growth and coalescence by microscopic necking or shearing. At certain conditions local strength inhomogeneities evoluate additionally to cavity-damage by martensitic transformation, dynamical ageing or recrystallization, grain growth and textural changes. Previous theories of ductile rupture $[1,2]$ and plastic stability $[3-6]$ do not involve such, likly nonlineare inhomogeneity evolutions expecially at superposed hardening and internal damage formation to an initial inhomogeneity. In the present study such effects for arbitrary strain-induced evolutions upon macroscopic neck development and ductility properties as rupture and local fracture strain are outlined in frame of an uniaxial longwavelength model modified by stress-triaxiality evolution within neck. Especially the important role of rate sensitive hardening and inhomogeneity rate, which for internal damage correlate to particle concentration and external pressure are considered. Classical predictions upon plastic stability are extended to this point of localized structural changes which result at critical conditions to a new "plastic metastable" state.

\section{Ductile rupture model}

During deformation internal damage forms by nucleation and growth of cavities at second particles which reduces effective cross-section $\Delta F_{c}>0$ locally. That may be superposed by developed axially strength changes either structural induced during necking or by inhomogeneous applied conditions leading to total inhomogeneity $\Delta f_{d}=\Delta F_{c} / F+\Delta \sigma / o$ where $\Delta \sigma>0$ denotes a local softening and $\Delta \sigma<0$ a local hardening. A strain-induced inhomogeneity evolution bejond a critical strain $\varepsilon_{0}>0$, i.e. plastic stability limit, may be general described by

$$
\Delta f=\Delta f_{o}+c \cdot\left(\varepsilon-\varepsilon_{0}\right)^{a}
$$


where $\Delta f_{0}$ is a pre-inhomogeneity and coefficient $a \geqslant 1$ and parameter $c$ denote rate of additional inhomogeneity evolution $\mathrm{c}>\mathrm{o}$ or degradation $\mathrm{c}<0$ if local hardening exceeds cavity damage. Uusually strengthening follows the power law

$\sigma=K \varepsilon^{n} \dot{\varepsilon}^{m}$

where $\mathrm{n}$ is the strain hardening coefficient, $\mathrm{m}$ the strain rate sensivity and $\varepsilon, \dot{\varepsilon}$ are the true strain and strain rate respectively resulting in $\Delta \sigma / \sigma=\Delta \mathrm{K} / \mathrm{K}$. For that and localized inhomogeneity formation, rate of local deformation development under uniaxial tension is described by the long-wavelength analyses [7]. Applying this procedure we derive in case of a linear inhomogeneity change $a=1$ and $n=0, \varepsilon_{0}=0$ at integers values $1 / m=1,2,3 \ldots$ for the true rupture strain the solution

$\varepsilon_{R}=-m \ln \left\{1-\left(1-\Delta f_{o}\right)^{1 / m}\left[1+\sum_{i=1,2,}^{i \leq p=1 / m} a_{i}^{p}(-c)^{i}-\exp (-A)\right]\left[1+\sum_{i=1,2}^{i \leq p=1 / m} a_{i}^{p}(-c)^{i}\left(1+\sum_{q=1,2}^{q=i} \frac{A^{q}}{q !}\right)\right]\right\}$

with the coefficients

$$
\alpha_{i}^{p}=(p)^{-i}[p \cdot(p-1)(p-2) \ldots \geq i] \leq 1 \quad a_{i}^{1}=1
$$

Here parameter A depends on macroscopic fracture initiation by coalescence of microscopic cavity-damage either occur at critical amount of local strain, $A=\hat{\varepsilon} / \mathrm{m}$, stress $\sigma_{\mathrm{f}}, A=\mathrm{P}_{\mathrm{f}} / \sigma_{\mathrm{f}} \cdot \mathrm{m}$ where $P_{f}$ the load at fracture or damage $\Delta f^{x}, A=\Delta f^{x} / c \cdot m$. In case of a nonlinear damage evolution $\alpha \gtrless 1$ at $\hat{\varepsilon}=\infty$ rupture strain is described by

$\varepsilon_{R}=-m \ln \left\{1-\left(1-\Delta f_{o}\right)^{1 / m}\left[1+\sum_{p=1,2, . .}^{p \leq 1 / m}\left(\begin{array}{l}1 / m \\ p\end{array}\right)(-c)^{p} m^{a} \Gamma_{(p \cdot a)}\right]\right\}$

for $a<1$ and

$$
\varepsilon_{R}=-m \ln \left\{1-\left(1-\Delta_{f o}\right)^{1 / m}\left[1+\sum_{p=1,2}^{p \leq 1 / m}\left(\begin{array}{l}
1 / m \\
p
\end{array}\right)(-c)^{p} m^{p \cdot \alpha}(p \cdot \alpha) !\right]\right\}
$$

for $\alpha=1,2,3$. . where $\Gamma_{(p \cdot a)}$ is the Gamma Function of order $p \cdot \alpha$ which mainly correct coefficients $a_{i}^{p}$ according eq. 3 . For superposed strain and strain rate sensitive hardening $n, m$ $>0$ and $\alpha=1$ we find for the engeneering strain

$$
\bar{\varepsilon}_{R}=E_{Z}^{1 / m}\left\{1-\left(1-\Delta f_{o}\right)^{1 / m} T_{T}\left[V_{Z}-\exp (\hat{\varepsilon} / m) \Phi_{Z}\right]\right\}^{-1 / m}-1
$$

where functions $V_{z}(c, m, z), \Phi_{z}(c, m, \hat{\varepsilon}, z)$ and $E_{z}\left(\varepsilon_{R}, z\right)$ depend additionally upon $z=n / m=1,2 \ldots$ . For $z, \hat{\varepsilon} \rightarrow \infty, \Delta f_{0}=0, c>0$ eq. 5 convergence to

$$
{ }^{\varepsilon_{R}}=s \cdot n-m \ln \left(T_{T} V_{R} C\right)
$$

with $\mathrm{s}=1$ similar as proposed by [7] for $\Delta \mathrm{f}_{\mathrm{o}}=$ const. in uniaxial case $\left(\mathrm{T}_{\mathrm{T}}=1\right)$ negleting $\mathrm{n}$ for necking. Eq. 6 may be used as approximation too for $\mathrm{z}<\infty$ where $\mathrm{s}<1$.Eqs. $3-6$ demonstrate that with increasing damage generation rate $c>0$ rupture strain is reduced more pronounced at large $m+n$, weak pre-inhomogeneities and delayed fracture onset, i.e. large $\Delta f x, \varepsilon_{f}$ and $a<1$. Woodfords [8] compilation $m$ vs. $\varepsilon_{R}$ modified to damage evolution shown in fig. 1 demonstrate the remaining essential effect of $m$ against $n$. But such universal relation for different materials does not exist. 

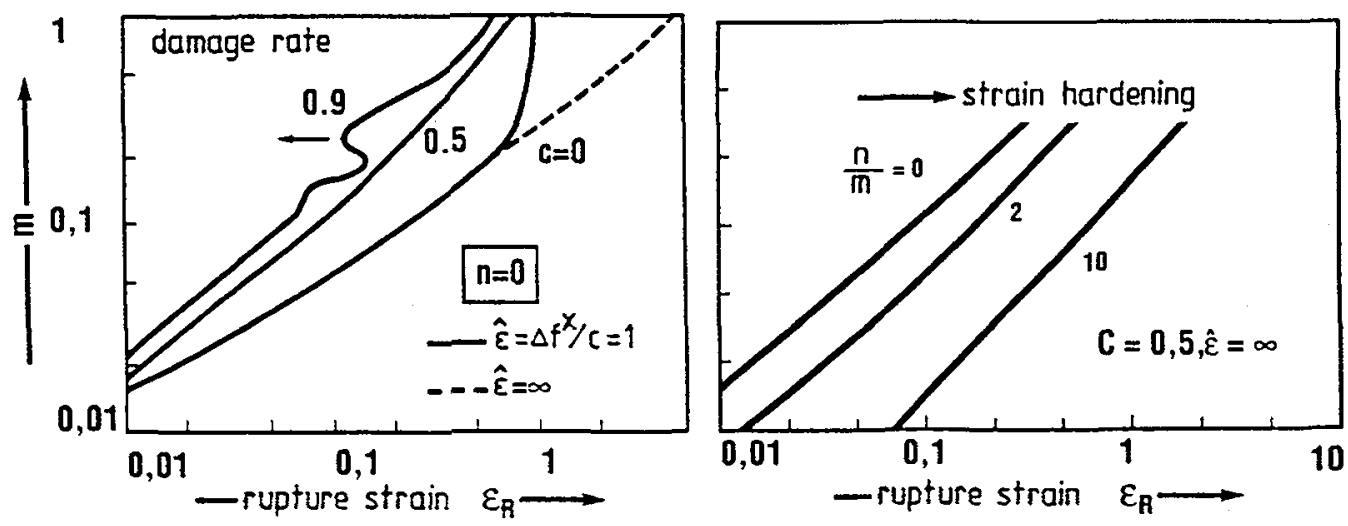

Fig. 1: Woodfords rupture strain diagram at damage generation $c>$ ofor $n=0$ and $a=1$

Uniaxial predictions have to be corrected by evoluated stress-triaxiality during necking which reduces mean effective stress $\sigma_{e}$ against uniaxial average stress $\sigma_{\mathfrak{u}}$, enhances interfacial particle debonding and cavity nucleation by increased hydrostatic tension $\sigma_{\mathrm{h}}$ and possible changes fracture initiation conditions. For small $\Delta f_{0}$ and unoched samples with axisymmetric cross-sections retard necking by $\sigma_{e}<\sigma_{u}$ usual exeeds particularly at small $\mathrm{m}$ where axial spread out of local deformation is restricted. Uniaxial models therefore underestimate real rupture strains without external pressure $p=0$ assymtotic. By such, triaxiality reflects a superposed hardening which reduces mean rate of damage development $\mathrm{C}_{\mathrm{T}}=\mathrm{C}_{\mathrm{e}}-\mathrm{C}_{\mathrm{h}}$ corrected by that enhance via increased hydrostatic tension $\sigma_{\mathrm{h}}-\mathrm{p}$. The first term is expressed by $\mathrm{C}_{\mathrm{e}}=\left(1-\mathrm{B}_{\mathrm{T}}\right) / \mathrm{B}_{\mathrm{T}} \varepsilon_{\mathrm{f}}$ with $\mathrm{B}_{\mathrm{T}}=\sigma_{\mathrm{e}} / \sigma_{\mathrm{u}}$ at fracture and the second $\mathrm{C}_{\mathrm{h}}=\mathrm{C}_{0} \varepsilon_{\mathrm{n}} \mathrm{T} / \varepsilon_{\mathrm{n}} 0$ depends upon triaxiality induced change in mean strain for cavity nucleation, thus $\left(\sigma_{h}-p\right) / \sigma_{e}$ for stress induced particle deponding or fracture $\sigma_{\text {normal }} \geq \sigma_{\mathrm{D}}$. The triaxiality evolution for nearly rigid-plastic conditions are given by the Bridgman analyses [9]

$$
B_{T}=1 /[(1+\eta) \ln (1+\eta)] ; \frac{\sigma_{h}}{\sigma_{e}}=1 / 3+\ln (1+\eta)-P / \sigma_{e}
$$

with the maximum hydrostatic tension $\sigma_{\mathrm{h}}$ at sample center. The ratio a/R $=2 \cdot \eta$ of minimum radius to the curvature of neck profile increases with strain $\varepsilon=2 \ln \left(a_{0} / a\right)$ by $n=K_{\eta}(\varepsilon-n)$ nearly independent on material properties $\mathrm{Kn}=0.38$ i.e. $\mathrm{Ce}=0.19\left(\mathrm{C}_{1}-\mathrm{n} / \hat{\varepsilon}_{\mathrm{f}}\right) /\left[1+0.19\left(\mathrm{C}_{1}\right)\right.$ $\left.\left.\mathrm{n} / \hat{\varepsilon}_{f}\right)\right]$ with $\mathrm{C}_{1}=\varepsilon_{\mathrm{f}} / \hat{\varepsilon}_{\mathrm{f}} \leq 1$ and $\mathrm{C}_{\mathrm{e}} \leq 1 / 4$ for $\mathbf{n} \geq 0$. Without neck profile measurements $\mathrm{n}(\varepsilon)$ required for real corrections, the function $T_{T}$ appeared in eq. 5 , using uniaxial particle deponding condition $(\varepsilon<n)$ may expressed by

$$
T_{T}=\beta_{e} \sigma_{e} / \sigma_{u}\left[1+\beta_{p} P / \sigma_{e}\right]
$$

where the coefficients $\beta_{e, p}$ are determind from measurements of pressure dependent increase in $\varepsilon_{R}$ or by rate of flow localization $\dot{\varepsilon}_{0} / \hat{\varepsilon}_{i}=f(\varepsilon, m) \dot{\varepsilon}_{i}=\hat{\varepsilon}$ which for an initial sinusoidale defect of wavelength $\lambda=c \cdot R$ related to $R$ is about [5],

$$
\dot{\hat{\varepsilon}}_{i}^{u} / \dot{\hat{\varepsilon}}^{T} \leq 1-\left(\frac{\pi}{4 \cdot c}\right)^{2}\left[1-3.25 \cdot m(\eta / c)^{2}\right]
$$

i. e. triaxiality retards necking pronounced at small $\mathrm{m}$ and neck-lengths as described eq. 2 too. Eqs. 5 - 8 predict a pressure dependence of rupture strain ratio 


$$
\begin{gathered}
\varepsilon_{R}^{p} / \varepsilon_{R}=1+K_{O} \ln \left(1+\beta_{p}^{\left.p / \sigma_{e}\right)=1+K_{O} \ln \varepsilon_{f}^{P} / \varepsilon_{f}^{o}}\right. \\
K_{O}^{-1}=\ln \left(T_{T} V_{K} C\right)^{o}-n / m \quad V_{K}=\sum_{i=1,2}^{i \leq p=1 / m} a_{i}^{P}(-C)^{i-1} \approx 1-(1-m) c \leq 1
\end{gathered}
$$

which assymtotes for $\mathrm{p} \rightarrow \mathrm{o}$ to the linear form

$$
\varepsilon_{R}^{P} / \varepsilon_{R}^{o}=1+K_{p} p / \sigma_{e} \quad, K_{p}=K_{O} \beta_{p}
$$

like as local fracture strain ratio

$$
\varepsilon_{f}^{p} / \varepsilon_{f}^{o} \simeq 1+\beta_{p} p / \sigma_{e}
$$

for $\Delta f^{x} \not p$ as found experimentally on various materials [10] and predicted by void-growth in principal tensile direction [11] too. Assuming a nucleation controlled increase in damage rate $\mathrm{C}_{\mathrm{u}} \sim \mathrm{K}_{2} \hat{\mathrm{f}}_{1, \mathrm{p}}$ with local particle volume concentration involved in deponding $\varepsilon_{\mathrm{n}} \leq \hat{\varepsilon}_{\mathrm{f}}$ which related to the total likely by $\hat{f}_{l, p} \simeq f_{p}^{t}, t=2 / 3$ otherwise a particle concentration dependence of $\varepsilon_{R}$ results

$$
\exp \left(\frac{n-\varepsilon_{R}}{m}\right) \simeq T_{T} V_{K} K_{2} f_{p}^{t} \simeq K_{R} C_{1} \varepsilon_{f}^{-1}
$$

where $\exp \left[\left(\mathbf{n}-\varepsilon_{R}\right) / m\right]$ is inverse related to fracture strain $\varepsilon_{f}$. Thus relation $\varepsilon_{R} v s$. $f_{p}$ becomes weaker at small $\mathrm{m}$ and $\mathrm{n}$ whereas $\varepsilon_{\mathrm{f}}$ only depend upon ratio of critical damage to deponding stress $K\left(\sigma_{D}\right)$ and volume concentration

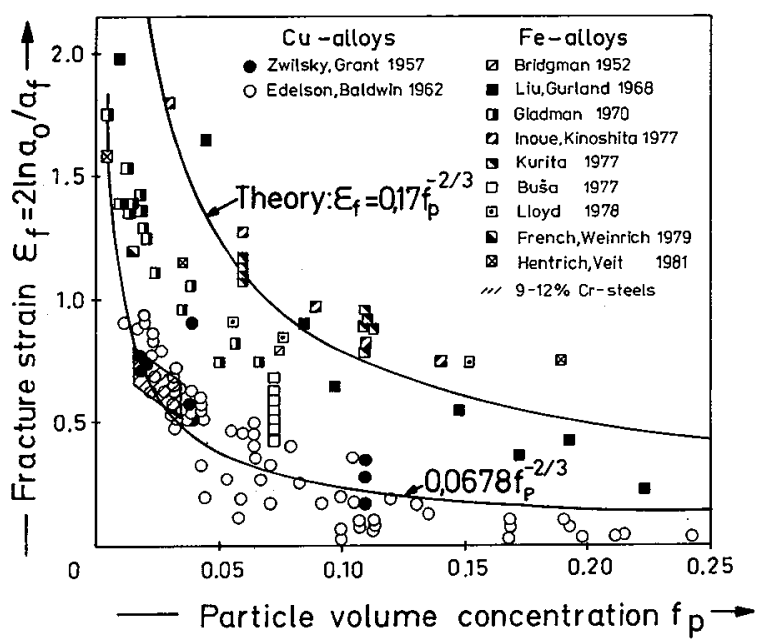

Fig: 2: Particle concentration depencence of fractrue strain 
which follows the basic trend of various materials for nearly $\mathrm{C}_{1} \Delta \mathrm{f}^{\mathrm{x}} / \mathrm{K}_{2}=$ const. $\simeq 0.05-0.17$, shown in fig. 2. The scatter visible describes deviations in $\Delta f^{\mathrm{x}}, \mathrm{K}$ by kind, form and size distribution of particles. Ductile rupture in accordance to model predictions and experiments seems therefore to result by mainly nucleation controlled damage formation whereby alined cavity-growth may reduce critical damage for coalescence and fracture initiation. The microscopic events of ductile rupture are as follows: At early necking cavities nucleate at large, elastic anisotropic particles which subsequent grow in principal tensile direction. This mainly nucleation controlled damage evolution step where nucleation continuous spreads to smaller particles mainly determine macroscopic ductility properties particularly at nonsuperplastic conditions of small $\mathrm{m}$. At a critical damage amount of about $\Delta \mathrm{f}^{\mathrm{x}}=0.1-0.3$ [12] alined cavities link up either by localized necking or shearing depending on triaxiality state, material properties and strain rate. This starts at the strong damage developed center (high $\left.\sigma_{n}\right)$ and progress radially under increasing triaxiality, strain rate gradient evolution, extensive microvoid nucleation at smaller particles and rotation of alined cavities leading preferential to cup-cone fracture. This coalescence step corresponds similar to dynamical fracture toughness situation and forms mainly the dimple fracture surface morphology. For viscouse fracture through localized shearing, fracture stress $\sigma_{f} \simeq \mu / 2 \pi d_{c} l / \varepsilon_{f}$ and parameter $A$ according eq. 3 depend on shear modulus $\mu$ and width, $d_{c}$, and total length, 1 , of micronecks at fracture. At small toughness $K_{i c}$ otherwise brittle fracture is prefered above $\sigma_{f} \approx K_{1 c} /(n / 2$ $\left.l_{c}\right)^{1 / 2}$ by propagation of cracks nucleated with a critical length $l_{c}$ corresponding to a characterized particle distance.

\section{Plastic metastability}

Plastic instability onset depends on considered initial defect type (mechanical or geometrical) and that growth criteria like area-reduction [4], defect-length, strain or strain rate which differ particularly at large m [13]. Considering now the local flow evolution and load change in case of an inhomogeneity increase and decrease. From eq. 3 we deduce for the local strain rate ratio at $n, \varepsilon_{0}=0$

$$
\dot{\varepsilon} / \dot{\varepsilon}_{i}=\left(1-\Delta f_{o}\right)^{1 / m} B_{a} \exp \left[-\left(\varepsilon_{i}-\varepsilon\right) / m\right]
$$

with the combined load change

$$
P / P_{o}=\frac{F_{q}}{\left(B_{a}\right)^{m}} \exp (-\varepsilon), \quad F_{q}=\frac{1-\Delta f_{d}}{1-\Delta f_{o}}
$$

, mainly characterized by the function $B_{\alpha}\left(m, c, \varepsilon_{i}\right)$ which for $\alpha=1$

$$
B=\sum_{p=0,1,2 \ldots}^{\leq p=1 / m}\left(\begin{array}{l}
1 / m \\
p
\end{array}\right)\left(-c \cdot \varepsilon_{i}\right)^{P}
$$

Fig. 3 demonstrate that behaviour at conditions $\mathrm{c}=0$ and $\mathrm{C} \gtrless 0$. For constant inhomogeneity load continuous drops and subsequent saturates where strain rate evolution becomes unbounded. Small load drops with ealier saturation result at small $\mathrm{m}$ and large $\Delta \mathrm{f}_{\mathrm{o}}$. A damage increase supress load drop and proceeds that saturation by enhanced flow localisation. An inhomogeneity decrease otherwise reduces neck growth and pronounces load drop in direction to homogeneous flow. Above a critical hardening rate, $\mathrm{c}<\mathrm{cx}<\mathrm{o}$ which correspond to a required critical ductility $\varepsilon^{\mathrm{x}} \leq \varepsilon_{\mathrm{f}}$, a transition from plastic instability to structural assisted stability occurs. After achive of homogeneous flow at $\varepsilon_{i}=\varepsilon^{x}$ instability process in an other local site which repeat so long as $\varepsilon_{\beta} \leq \varepsilon_{f}$. This plastic metastable state therefore is characterized by stochastic flow through structural pulsed micro-neck formation and 
resolution involving plastic instability to stability transitions. That increases rupture strain $\varepsilon \leq \varepsilon_{\mathrm{f}}$ assisted by the increased fracture strain through diminished cavity-nucleation via retard hydrostatic tension evolution. Thus plastic metastability might by a suited mean for development of ductile metals whith large potential particularly at small $\mathrm{m}$ and $\mathrm{n}$. However strong hardening within neck-zone is required here which might be result by strain or stress induced martensitic transformation, glide band hardening or dynamical ageing under certain conditions. Considering now the load serrations by plastic metastability. The critical strain $\varepsilon^{x}$ (cx) defining load frequency $\mathrm{v}=1 / \varepsilon^{\mathrm{x}}$ results from eq. 3 for $\varepsilon_{\mathrm{i}}=\varepsilon^{\mathrm{x}}$ with the lower bound for $\mathrm{cx}$ $\leq-\Delta f_{o} /\left(1-\Delta f_{0}\right)$ at $\varepsilon^{x} \leq \infty$. The maximum load change against homogeneous flow at instable to stable transition occurs at

$$
\varepsilon_{T}^{x}=\frac{m}{-c} \frac{\left(1-\Delta f_{a}\right)}{B^{x}} \sum_{p=1,2,}^{\leq p=1 / m}\left(\begin{array}{c}
1 / m \\
p
\end{array}\right)(-c)^{p}\left(\varepsilon_{T}^{x}\right)^{p}
$$

with $B^{x}=B$ at $\varepsilon_{i}=\varepsilon_{T}$ which gives $\varepsilon_{T} \simeq w \varepsilon^{x}$ with the coefficient $w\left(m, \Delta f_{0}\right) \approx 0.5$. Introducing this in eq. $13 \mathrm{~b}$ we have for the relative load change aggainst homogeneous flow

$\Delta P / P_{h}=F_{q} /\left(B^{x}\right)^{m}-1$

which gives for $\mathrm{m}=1$

$$
\Delta P / P_{h} \simeq F_{q} /\left(1+w \frac{c}{v}\right)-1
$$

valid also for $\mathrm{m}<1$ as approximation, whereby for the frequency the relation

$v \simeq\left\{\ln / P_{h} P_{i} F_{q}\right\}^{-1}$ may hold. Thus load change $\Delta P / P_{h}>0$ introduced by local flow increases with increasing frequency and decreasing strain rate sensivity. At superplastic and irradiation conditions small $\Delta \mathrm{P} / \mathrm{P}_{\mathrm{h}} \rightarrow \mathrm{O}$ results indicating that local structural changes can not by decide from homogeneous here because of dominant axially spread of deformation. Large load changes occur which reflect directly structural changes. At small $\mathrm{m}$ otherwise frequency and amplitude $\Delta \mathrm{P} / \mathrm{P}_{\mathrm{h}}$ of serrations in addiation to $\mathrm{m}$ mainly quantify local structural changes.

\section{References}

[1] A. W. Thompson, M. F. Ashby; Scripta Met. 18 (1984) 127

[2] Mc. Clintock; J. Appl. Mech. Trans ASME, Ser. E, 35 (1968)363

[3] M. Considérs; Ann. des Ponts et Chaussées, (1985) 574

[4] E. W. Hart; Acta Met. 15 (1967)351

[5] J. W. Hutchinson and K. W. Neale; Acta Met. 25 (1977) 839

[6] J. J. Jones and B Baudelet; Acta Met. 25 (1977) 43

[7] A. K. Ghosh, Acta Met. 25 (1977) 1413

[8] D.A. Woodford, Trans. Am. Soc. Metals 62 (1969) 291

[9] P. W. Bridgman; Trans. ASM 32 (1943) 553

[10] A. Brownrigg, W. A. Spitzig, O. Richmond, D. Teirlinck and J. D. Embury; Acta Met. 31 (1983) 1141

[11] J. R. Rice and D. M. Tracey; J. Mech. Phys. Solids 17 (1983) 201

[12] R. Becker; J. Mech. Phys. Solids 35 (1987) 577

[13] J. J. Jonas, R. A. Holt and C. E. Coleman; Acta Met. 24 (1976) 911 

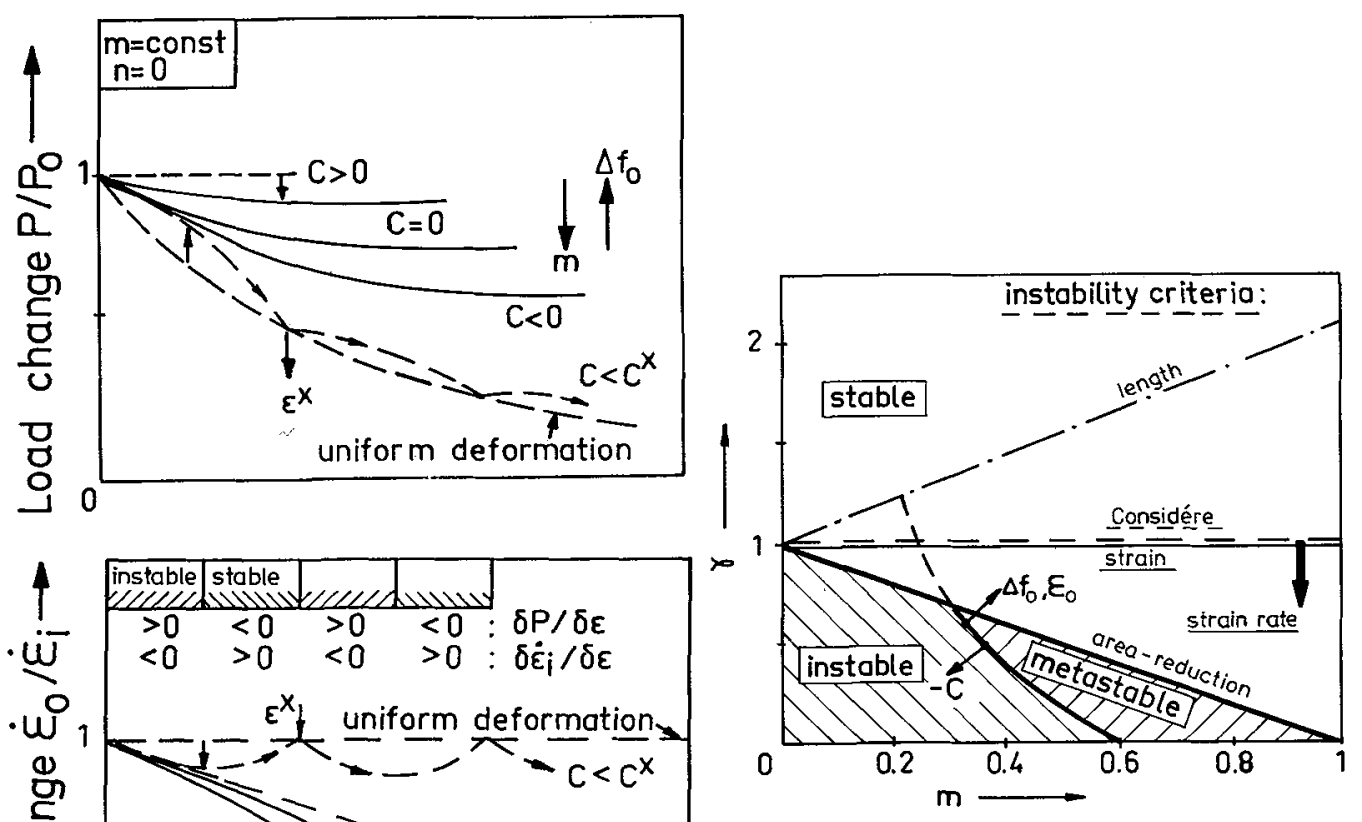

Fig. 4: Phase diagram for tensile flow at $\mathrm{c} \leq \mathrm{o}$

Fig. 3: load and strain rate change at local inhomogeneity generation $\mathrm{c} \leqslant \mathrm{o}$. 